\title{
Analysis of the strange phenomenon and optimization to connecting rod wheeled robot
}

\author{
Xiang $\mathrm{Hu}^{1, \text { a }}$, Yu-jun Wang ${ }^{2, b}$, Can Fang ${ }^{3, \mathrm{c}}$
}

${ }^{1,}$ School of Computer and Information Science, Southwest University, Chongqing,China and school of computer science and engineering, Chongqing Three Gorges University , Chongqing, China

${ }^{2,3}$ School of Computer and Information Science, Southwest University, Chongqing, China

a723765786@qq.com, b774872400@qq.com, '839147@qq.com

Keywords: Link wheel structure; adaptive topography; strange phenomenon

\begin{abstract}
This article is about optimization of connecting rod wheeled robot, connecting rod wheeled robot platform can be well adaptive terrain, on regular ground velocity structure near wheeled platform. On the road barriers, independent walking mode switching, efficient obstacle. In this paper, the structural of connecting rod wheeled robot platform will be analysised, According to the strange phenomenon were analyzed and resolved, Its performance is optimized. It is proved by experiments that the conclusion of this paper.
\end{abstract}

\section{Introduction}

Robot in this paper is designed as a connecting rod type structure, this structure can achieve the control is simple and high efficiency of obstacle, but it exist strange phenomenon. The design idea is to the same side of the wheel in the same position through the passive joints ${ }^{[1]}$ connecting them as shown in Fig 1.

The walking mechanism of connecting rod wheeled robot is connected to the connecting rod by a plurality of passive joint. The overall structure ${ }^{[2]}$ of the four wheels as the walking mechanism robot as shown in Fig 2. The structure belongs to

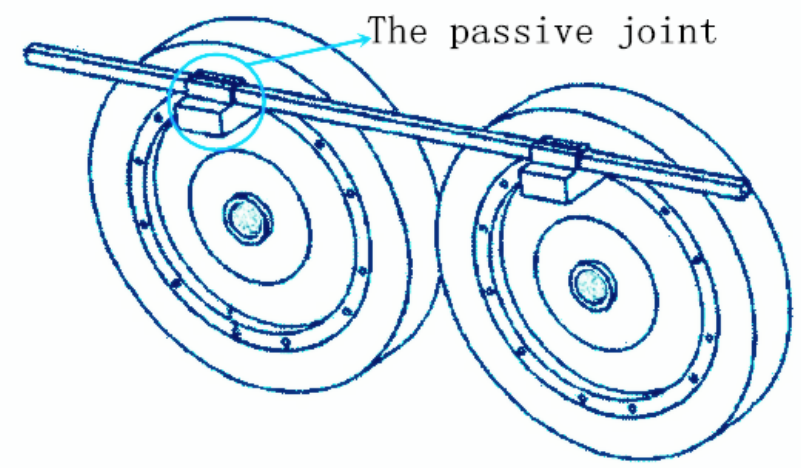

Fig 1 passive joints

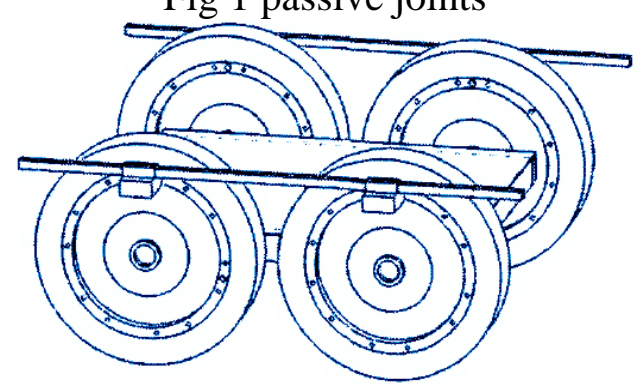

Fig 2 overall structure

The parallel mode and has good stability.In structured terrain its effectiveness is equivalent to the ordinary wheel and the center of gravity is kept constant.The robot with the geometric structure dimensions as shown in Fig 3.The center of the front and rear wheels are Ofront and Oback,The wheel radius is R,The connection point of front wheel and the connecting rod is Pfront, The rear 
wheel and the connecting point of the connecting rod is Pback. Eccentricity is the distance from Pfront to Pback. Let Ofront-Pfront=Oback-Pback=a, The maximum height to Cross the barrier is $\mathrm{R}+2 \mathrm{a}$.

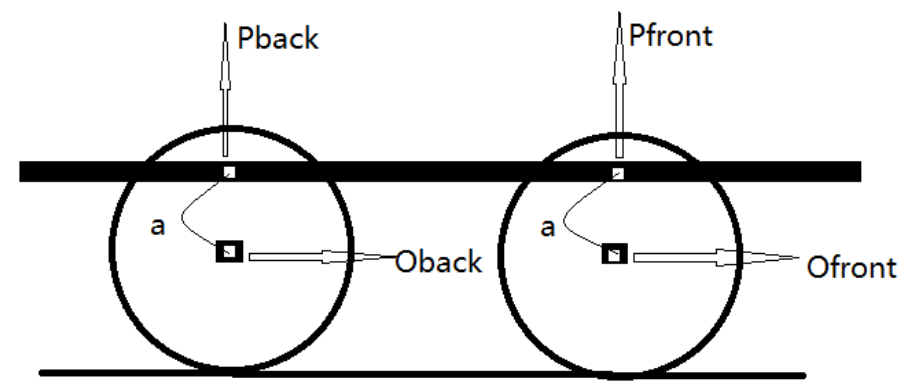

Fig 3 geometric structure

\section{Analysis of it strange phenomenon and the optimization scheme}

The strange phenomenon ${ }^{[3,4]}$ is defined as: The wheels of robot occur the phenomenon of clamping as the connecting rod position abnormalities. As shown in Fig 4,the angle between the rod and the ground is $\theta \in\left(0^{\circ}, 90^{\circ}\right)$, The connecting rod is a rigid material, the distance between Connect points will remain unchanged, The motor will be blocked, Robots appear strange phenomenon. When the center point of the wheels and the connection point of the connecting rod and the wheels are in the same line. As shown in Fig 5, $O_{1}$ and $O_{2}$ and $\mathrm{M}$ and $\mathrm{A}$ are in the same line. If the

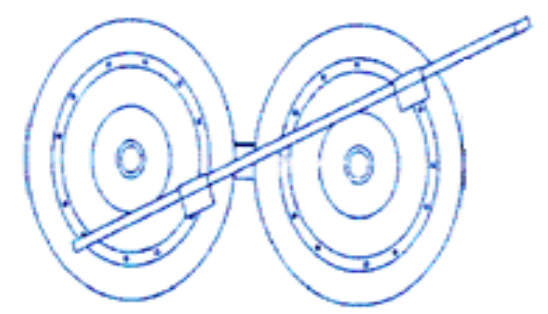

Fig 4 strange phenomenon

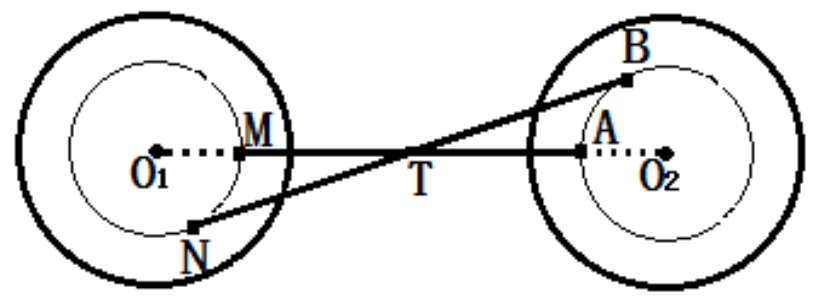

Fig 5 The 4 points collinear

speed of wheels is inconsistency, strange phenomenon will occur. $M$ will be transferred to the $\mathrm{N}$ point in time, $\mathrm{A}$ will be transferred to $\mathrm{B}$, The connecting rod $\mathrm{MA}$ around a point $\mathrm{T}$ to $\mathrm{NB}$ and $\mathrm{MA}=\mathrm{NB}$, One end of the connecting rod is above the line $O_{1} O_{2}$ and the other end of the connecting rod is under the line $O_{1} O_{2}$ like NB when strange phenomenon occur. If $O_{1}$ and $O_{2}$ and $\mathrm{M}$ and $\mathrm{A}$ are not in the same line, As shown in Fig 6. Arc HK is the circular arc to A as the center of a circle and the connecting rod MA as the radius. If the strange phenomenon will occur, The connecting rod was changed from MA to NA and The angle ${ }^{[5,6]}$ between the MA and NA is $\theta \in\left(0^{\circ}, 90^{\circ}\right)$. So there are:

$$
\left\{\begin{array}{l}
O_{1} M=O_{1} N \\
M A=N A
\end{array}\right.
$$

By the formula (1): strange point can only N, Due to the MA and is always constant, if M can be transferred to N, MA is to break the prerequisites for the constant in the transfer process, so that 
the $\mathrm{M}$ point can not be converted to $\mathrm{N}$. Therefore this assumption does not hold. If $\mathrm{O}_{1}$ and $\mathrm{O}_{2}$ and $\mathrm{M}$ and $\mathrm{A}$ are not in the

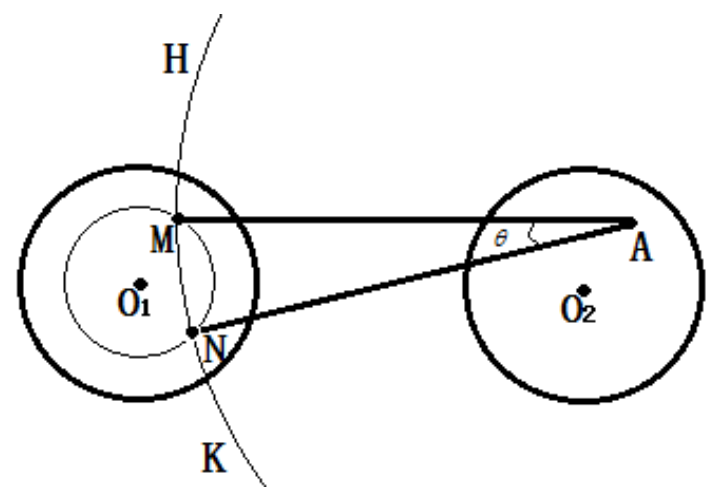

Fig 6 the 4 points different line

same line, strange phenomenon will not occur. To avoid the strange phenomenon has not occurred, The design of the robot is the connecting rod connect three wheels on each side. As shown in Fig 7.

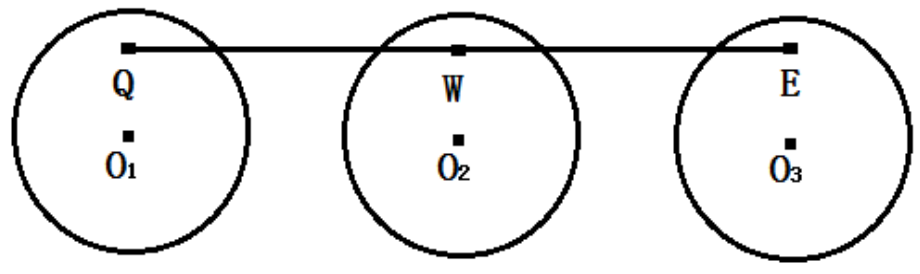

Fig 7 Structure optimization design

The centers of circles are $O_{1}, O_{2}$ and $O_{3}$, The connecting rod connect the three wheels at $\mathrm{Q}, \mathrm{W}$ and E. This design will completely avoid strange phenomenon. Assuming that this design will appear strange phenomenon, also is only possible the $O_{1}, O_{2}, O_{3}, \mathrm{Q}, \mathrm{W}$ and $\mathrm{E}$ are in the same line. This will be the three wheels are divided into two parts to analysis, as shown in Fig 8. The first part is wheel $O_{1}$ and wheel $O_{2}$,

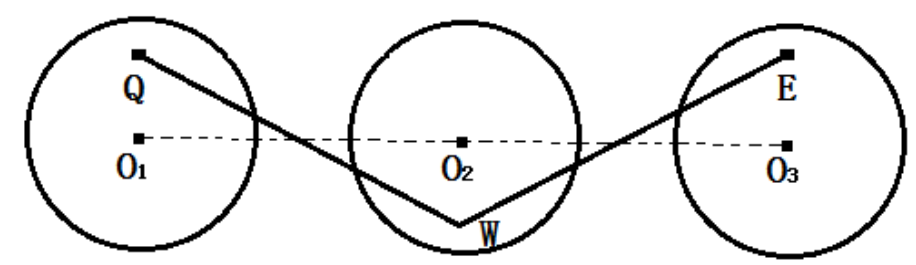

Fig 8 Structure optimization analysis

Through the conclusion of the strange phenomenon, the part of the connecting rod is bound to the QW in Fig 8 state. The second part is wheel $O_{2}$ and wheel $O_{3}$, Similarly, This part of the connecting rod is bound to the WE state in Fig 8. The connecting rod is a bending state in Fig 8.It does not occur because the connecting rod is rigid. So this structure will completely avoid strange phenomenon.

\section{Experimental analysis}

Through the above theory to the robot, the robot system was implemented, with STM32 as the main control chip, the two aluminum alloy as The connecting rod. According to 6 DC motors and 3 L298N drive modules to achive the robot ${ }^{[7,8,9]}$.It climb obstacle (step) on the front and side views are shown in Fig 9 and Fig 10. 


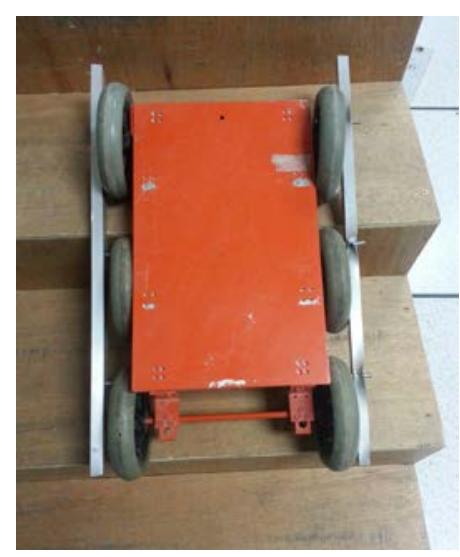

Fig 9 front view

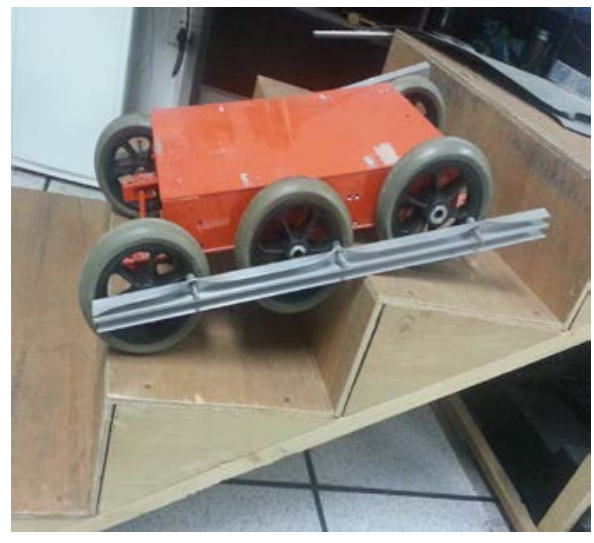

Fig 10 side view

The experiment shows that: The connecting rod wheeled robot with three wheels on each side can not appear strange phenomenon by climbing and walking, According to the 100 times obstacle terrain and 100 times regular terrain walking experiments, experimental results datas are listed in the following table 1 :

Table 1: Robot walking Situation

\begin{tabular}{|c|c|c|c|}
\hline $\begin{array}{l}\text { Robot walking } \\
\text { Situation }\end{array}$ & normal times & $\begin{array}{l}\text { strange phenomenon } \\
\text { times }\end{array}$ & Normal probability \\
\hline obstacle terrain & 100 & 0 & $100 \%$ \\
\hline regular terrain & 100 & 0 & $100 \%$ \\
\hline $\begin{array}{l}\text { Average } \\
\text { Speed }\end{array}$ & $\begin{array}{l}\text { Ordinary wheeled } \\
\text { platform }(\mathrm{m} / \mathrm{s})\end{array}$ & $\begin{array}{l}\text { Connecting rod } \\
\text { wheeled robot }(\mathrm{m} / \mathrm{s})\end{array}$ & $\begin{array}{l}\text { Loss of efficiency } \\
\text { By the connecting } \\
\text { rod }\end{array}$ \\
\hline obstacle terrain & 0.6 & 0.56 & $7 \%$ \\
\hline regular terrain & 0.95 & 0.91 & $5 \%$ \\
\hline
\end{tabular}

\section{Summary}

This article has carried on the analysis to the strange phenomenon in the process of connecting rod wheeled robot, By improving the structure of the robot, completely avoid the strange phenomenon, enhance its stability; It will have great prospect.

\section{Reference}

[1] Peng Zhang, Guoyin Wang, Chunmei Tao, et al. The program code similarity measure method based on Rough Set[J]. Journal of Chongqing University of Posts and Telecommunications (NATURAL SCIENCE EDITION), 2008, 20 (6): 737 741.

[2] Junke Li. wheel ski style robot design and analysis [D]. Chongqing: Southwestern University, 2013

[3] Xiaoyun Xu, Guozheng Yan, Guoqing Ding. Research on micro six legged robot and its tripod gait[J]. Optics and precision engineering, 2002,10 (4): 392 396. 
[4] Xingjun Tan. The eccentric wheel leg of six legged robot design and analysis [D]. Chongqing: Southwestern University, 2011

[5] Weigang Song. Robotics: kinematics and dynamics and control [M]. Beijing: Science Press, 2007:32-39.

[6] Zhiqiang Hou, Han Chongzhao. The visual tracking technology [J]. Acta automatica Sinica, 2006, 32 (4): 603 617.

[7] A.P.Englbrecht and A.Ismail.Training Product Unit Networks.Stability and Control:Theory and Applications,2(1-2):59-74,1999.

[8] U.Aickelin,P.J.Bentley,S.Cayzer,J.kim,and J.McLeod.Danger Theroy:The Link between AIS and IDS? In Proceedings of Second International Conference on Aritficial Immune Systems,Pages 147-155,2003.

[9] Rescue Robot Demonstrated for Mine Disasters[OL].http://crasar.csee.usf.edu. 